\title{
A concept of active mount for space applications
}

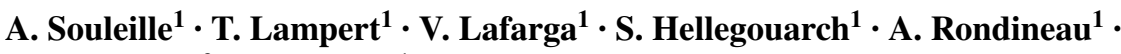 \\ G. Rodrigues ${ }^{2} \cdot$ C. Collette ${ }^{1}(1)$
}

Received: 26 October 2016 / Revised: 9 October 2017 / Accepted: 25 October 2017 / Published online: 30 October 2017

(C) CEAS 2017

\begin{abstract}
Sensitive payloads mounted on top of launchers are subjected to many sources of disturbances during the flight. The most severe dynamic loads arise from the ignition of the motors, gusts, pressure fluctuations in the booster and from the separation of the boosters. The transmission of these dynamic forces can be reduced by mounting payloads on passive isolators, which comes at the expense of harmful amplifications of the motion at low frequency due to suspension resonances. To bypass this shortcoming, this paper presents a novel concept of active mount for aerospace payloads, which is easy to install, and meets two objectives. The first one is a high damping authority on both suspension resonances and flexible resonances without compromising the isolation and large stability margins of the closed loop system due to the collocation of the actuator and the sensor. The second one is a broadband reduction of the dynamic force transmitted to the payload, which was achieved in terms of $16 \mathrm{~dB}$. The concept is presented in the first part of the paper and studied numerically and experimentally on a single degree of freedom isolator. A commercial isolator has been chosen for the purpose of the demonstration. The second part of the paper is dedicated to experimental validations on multi-degree of freedom scaled test benches. It is shown that the force feedback allows damping of both suspension and flexible modes (first and second modes,
\end{abstract}

$\checkmark$ C. Collette

ccollett@ulb.ac.be

G. Rodrigues

goncalo.rodrigues@esa.int

1 BEAMS Department, Université Libre de Bruxelles, 50, av. F.D Roosevelt, 1050 Brussels, Belgium

2 ATG-Europe B.V at European Space Agency, ESTEC, Paris, France respectively), and significantly reducing the force transmitted in some broad frequency ranges.

Keywords Launcher disturbances · Vibration damping · Active mount

\section{Introduction}

Since the world's first satellite has been launched nearly 60 years ago, the technology of launchers and satellites has considerably improved. While in the early days, satellites were mounted on rigid supports, modern and more sensitive satellite equipments and instruments require a better isolation from launcher disturbances [1]. To this purpose, passive suspensions have been progressively introduced. Mounting the satellites on passive suspensions produces a filtering of high-frequency vibrations [2-4]. The softer the suspension, the better the isolation.

However, this comes at the expense of large and harmful amplifications of the lower frequency suspension modes [5], and raises concerns of strength and clearance issues because of the larger compliance. This is a first fundamental limitation of passive isolation [6].

The amplification at resonance can be reduced by increasing the damping in the isolator. However, this comes in turn at the expense of a reduction of the isolation at high frequency. This is a second fundamental limitation of passive suspensions.

The only way to bypass the shortcoming above and simultaneously eliminate the resonance response of the suspension mode while avoiding a degradation of the attenuation at high frequencies is to use active vibration isolation systems. They do not show such amplifications at low frequencies and are capable of an overall damping performance. Considerable 
efforts on active [7-10] and semi-active vibration isolators $[11,12]$ have been carried out. However, measurement noise and stability concerns of the closed loop system have often hindered performance, or the final design of the isolator exhibited excessive mass.

An example of flight-proven vibration isolation system is the CSA Softride [2,3]. It consists of modules which can be installed for example around a ring, Fig. 1, and provides attenuations which can be up to 10 and $20 \mathrm{~dB}$ above the frequency of a suspension mode which can be tuned to between 25 and $500 \mathrm{~Hz}$. While very attractive on the grounds of its modularity and performance, the isolation is obtained at the cost of a large magnification of the launcher vibration around its main resonance, where the payload is bouncing on the stiffness of the suspension [2]. This feature is inherent to passive systems.

This paper presents a novel concept of active mount for aerospace payloads which uses an architecture similar to the Uniflex Softride, and combines it with active control

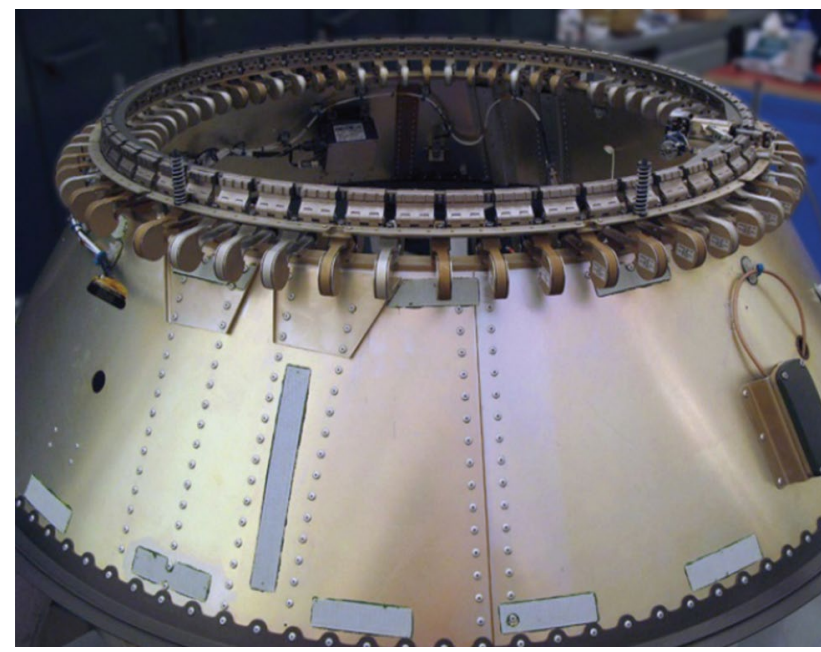

Fig. 1 Moog's Softride technology. Photo courtesy Sierra Nevada Corporation and Moog [3] capability. This approach reduces the magnification due to the first structural resonances of the suspension mode and preserves the vibration attenuation at high frequencies. It offers several advantages, including ease of mounting, high damping authority on both suspension resonances and flexible resonances without compromising the high-frequency isolation and large stability margins of the closed loop system.

The paper is organized as follows. Section 2 presents the concept of active isolator, along with a number of claimed advantages of this concept, Sect. 3 studies numerically and experimentally the performance of the isolator using a single d.o.f. system, Sect. 4 extends the concept to a flexible payload mounted on three isolators. In Sect. 5, a flexibility is introduced below the isolator.

\section{Proposed concept of active isolator}

The proposed concept of active isolator is composed of a compliant metallic structure with an integrated sensor and a piezoelectric actuator. An example of this configuration is shown in Fig. 2. As for flight-proven isolator, it consists of an elliptic metallic structure that plays the role of suspension. Additionally, a piezoelectric stack actuator has been placed inside the structure, along the horizontal axis of the ellipse. The stack is further divided into two parts, respectively used as actuator and as force sensor, and both constitute a perfectly collocated pair.

In each mount, the actuator will be driven only using the signal from the force sensor of the same mount. Both suspension resonances and payload resonances are actively damped by applying viscous damping forces obtained by integrating the signal from the force sensors [13]. Such control strategy, known as integral force feedback (IFF), has been extensively discussed in $[14,15]$. In the proposed configuration, it can also be noticed that the softening effect inherent to force control is limited by the metallic suspension, which will also continue to
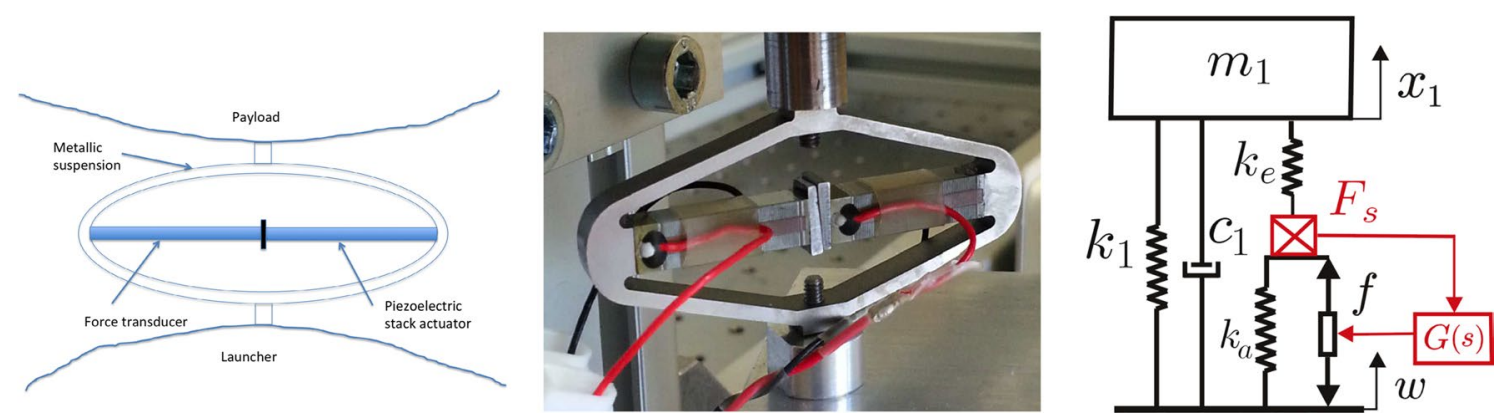

Fig. 2 From left to right: concept of active isolator; picture of an APA $100 \mathrm{M}$ from Cedrat Technologies used for the experiments (one piezoelectric stack is used as force sensor and the other one is used as actuator); simplified model of a one d.o.f. payload mounted on such isolator 
work as a passive isolator in case of failure of the piezoelectric stack.

The advantages of this mount are:

1. Modularity: The number and position of mounts will depend on the mass and inertia of the payload and the specific mechanical environment.

2. The elliptical shape is working both as a suspension spring and as a mechanical amplifier for increasing the actuator range.

3. Each unit is stand-alone (does not require any additional sensor).

4. The control architecture use decentralized feedback loops in each active mount, which offers excellent stability margins.

Although the full design of the suspension will require a complete study, the objective of this preliminary work is to test experimentally the concept using a commercial isolator. To this purpose, several setups of increasing complexity have been developed: one d.o.f payload mounted on one isolator, flexible payload mounted on three isolators and the same structure mounted on a flexible launcher are presented, respectively, in Sects. 3, 4 and 5.

\section{Single degree-of-freedom isolator}

Figure 2 shows a picture of the amplified piezoelectric stack used for the experiments. It is an APA $100 \mathrm{M}$ from CedratTechnologies, where the piezoelectric actuator has been divided into two parts: one is used as actuator, and the other one is used as force sensor. A small mass has been mounted on the actuator to represent a single d.o.f. payload. A simplified sketch of the system is shown in Fig. 2 (right). In this model, $k_{1}$ represents the stiffness of the metallic suspension when the stack is removed; $k_{a}$ is the stiffness of the actuator; $k_{e}$ is a stiffness used to adjust the pole of the isolator; $w$ is the motion imposed by launcher; $F_{s}$ is the force sensor; $f$ is the actuator force; $F$ is the external force applied to $m_{l} ; G(s)$ is the controller; $x_{1}$ is the motion of the payload; $c_{1}$ is a viscous damper used to match experimental results. The dynamic equation of the system is

$$
\begin{aligned}
m \ddot{x}_{1}= & \left(-k_{1}-\frac{k_{e} k_{a}}{k_{e}+k_{a}}\right) x_{1}+\left(k_{1}+\frac{k_{e} k_{a}}{k_{e}+k_{a}}\right) w \\
& -c_{1} \dot{x}_{1}+c_{1} \dot{w}+F+\left(\frac{k_{e}}{k_{e}+k_{a}}\right) f .
\end{aligned}
$$

The expression of the force measured by the force sensor is

$$
F_{s}=\left(-\frac{k_{e} k_{a}}{k_{e}+k_{a}}\right) x_{1}+\left(\frac{k_{e} k_{a}}{k_{e}+k_{a}}\right) w+\left(\frac{k_{e}}{k_{e}+k_{a}}\right) f \text {, }
$$

and the control force is given by

$f=F_{s} \cdot G(s)$

where $G(s)=g / s$ and $g$ is the control gain.

Figure 3 shows the matrix of transfer function from input $(w, f, F)$ to output $\left(F_{s}, x_{1}\right)$ in open loop (blue curves) and closed loop (dashed red curves). The following numerical values have been used for the simulation: $m=1 \mathrm{~kg} ; k_{e}=4.8 \mathrm{MN} / \mathrm{m} ; k_{1}=960 \mathrm{kN} / \mathrm{m} ; k_{a}=65 \mathrm{MN} / \mathrm{m}$; $c_{1}=10 \mathrm{Ns} / \mathrm{m}$. These values have been chosen to have a matching between the theoretical and the experimental open loop transfer function $F_{s} / f$. The gain has been arbitrarily chosen as $g=1000 \mathrm{rad} / \mathrm{s}$ for illustrating the performance of the closed loop system.

Firstly, it can be noticed on the transmissibility that the resonance peak is almost critically damped, without compromising the high-frequency passive isolation for frequencies higher than the resonance of the suspension. Secondly, the degradation of the compliance induced by the feedback operation is limited at $l / k_{1}$ as anticipated. Thirdly, the fraction of the force transmitted to the payload that is measured by the force sensor is reduced not only at the resonance, but also in a broad frequency range at low frequencies.

Figure 4 compares the theoretical (solid curves) and experimental (dashed curves) closed loop transfer functions between the sensor and the actuator obtained for various values of the control gain. It clearly shows a high efficiency of the isolator and a good match between the model and the experiment. The experimental data are further compared with the model in Fig. 5, which shows the trajectory of the closed loop poles in the complex plane. The experimental points have been obtained by fitting the measured closed loop transfer function.

The little mismatch between the theory and the experiment is attributed to the fit algorithm which has been used to calculate the value of the closed loop poles, because highly damped poles are difficult to fit precisely.

\section{Flexible payload mounted on three isolators}

The second setup considered consists of a heavier payload, mounted on a set of three isolators. The payload consists of two masses, connected through three flexible blades with a tunable length. The setup is shown in Fig. 6, along with a simplified sketch showing only the vertical d.o.f. of the payload, mounted on one isolator.

The flexible resonance of the payload in the vertical direction has been tuned around $65 \mathrm{~Hz}$. It corresponds to the resonance of $m_{2}$ while $m_{1}$ is blocked. The first six mode shapes and corresponding resonance frequencies are shown in Fig. 7. The setup has been mounted on a passive 

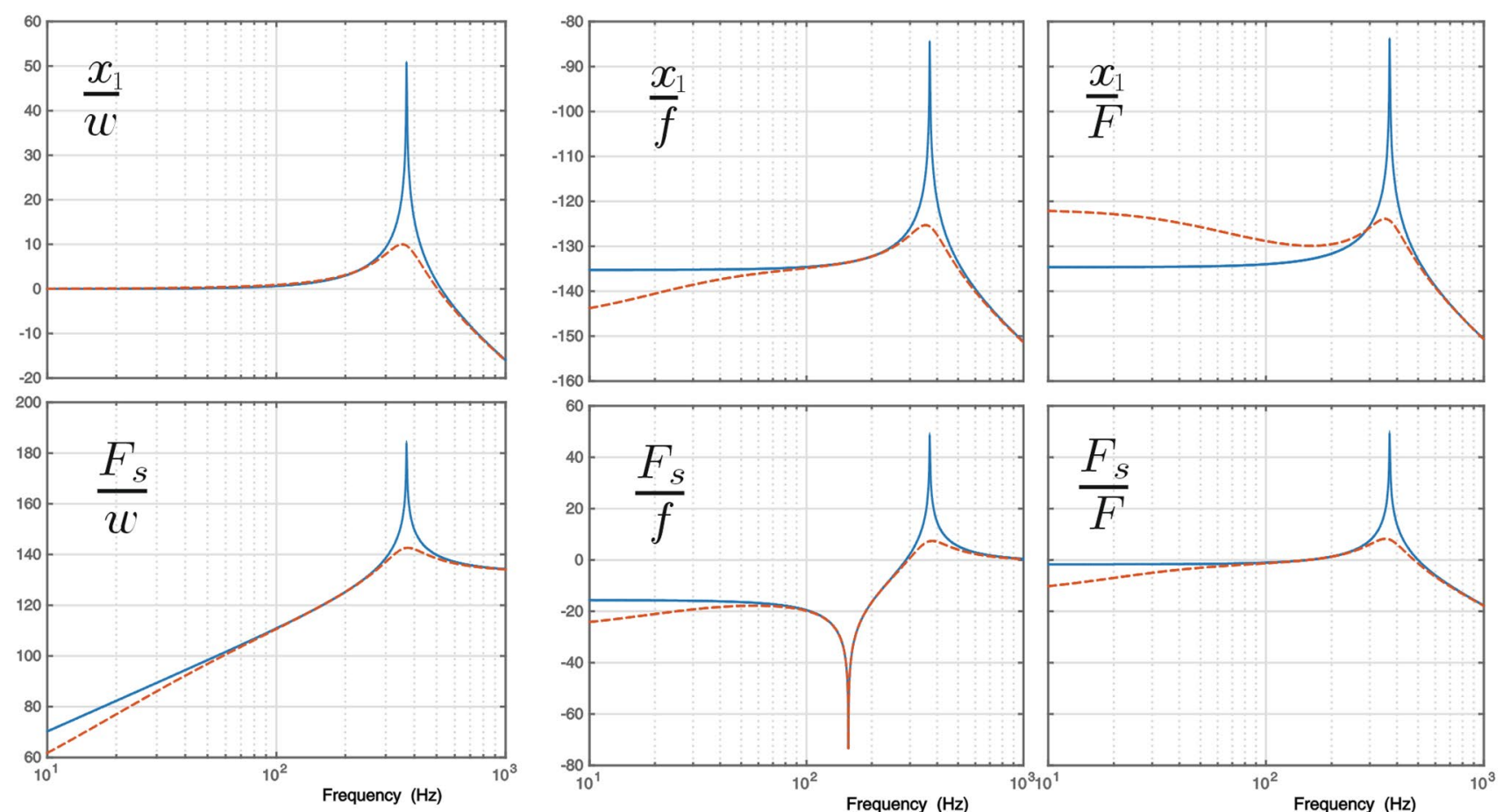

Fig. 3 Matrix of transfer functions from input ( $w, f, F)$ to output $\left(F_{s}, x_{1}\right)$ in open loop (blue curves) and closed loop (dashed red curves)

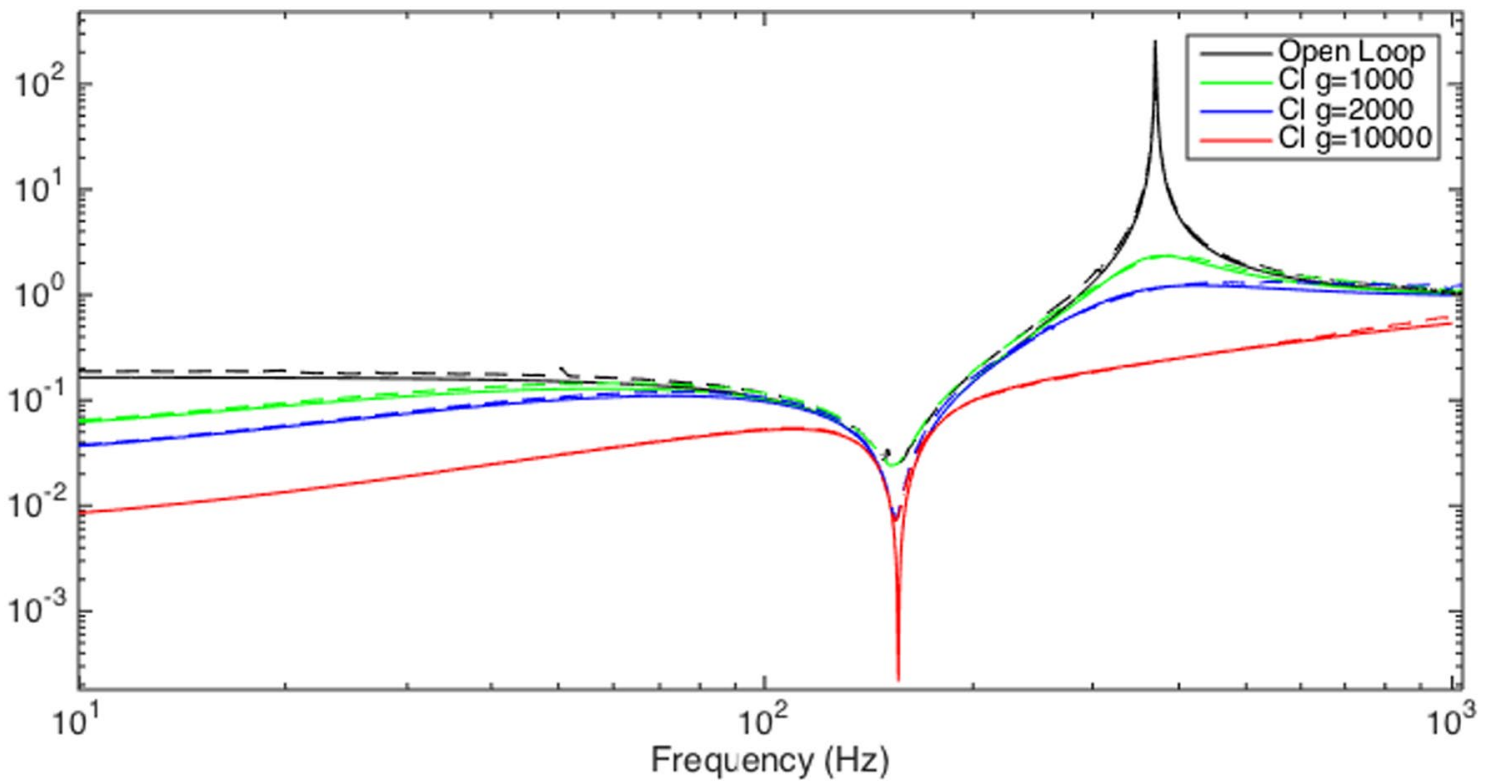

Fig. 4 Open loop and closed loop transfer function between the actuator and the force sensor. Comparison between the model (solid curve) and the experiment (dashed curve)

optical table. A shaker has been mounted on the table top besides the setup in order to excite it from the base as for the launcher.

Two test campaigns have been conducted: one when the shaker excites the table in the vertical direction, and one when the shaker excites the table in the horizontal direction. For each case, a random voltage is applied to the shaker during $1 \mathrm{~min}$. For both campaigns, decentralized control loops have been used, as explained in Sects. 1 and 2. Typical experimental results are shown, respectively, in Figs. 8 and 


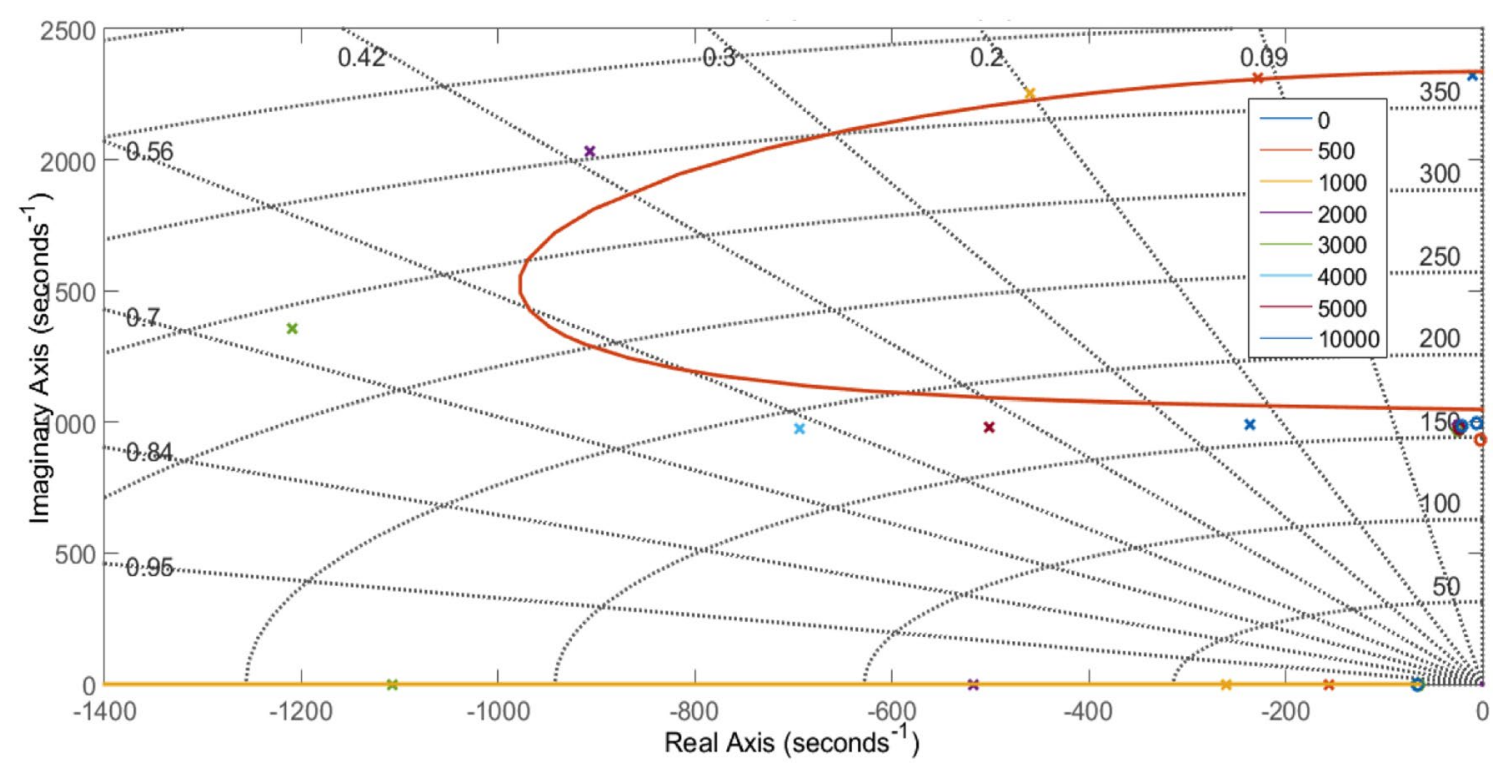

Fig. 5 Single d.o.f. system. Comparison between the theoretical (solid curve) and the experimental (crosses) root-locus

Fig. 6 Right: picture of the experimental setup. It consists of a flexible payload mounted on a set of three isolators. Left: simplified sketch of the setup, showing only the vertical direction

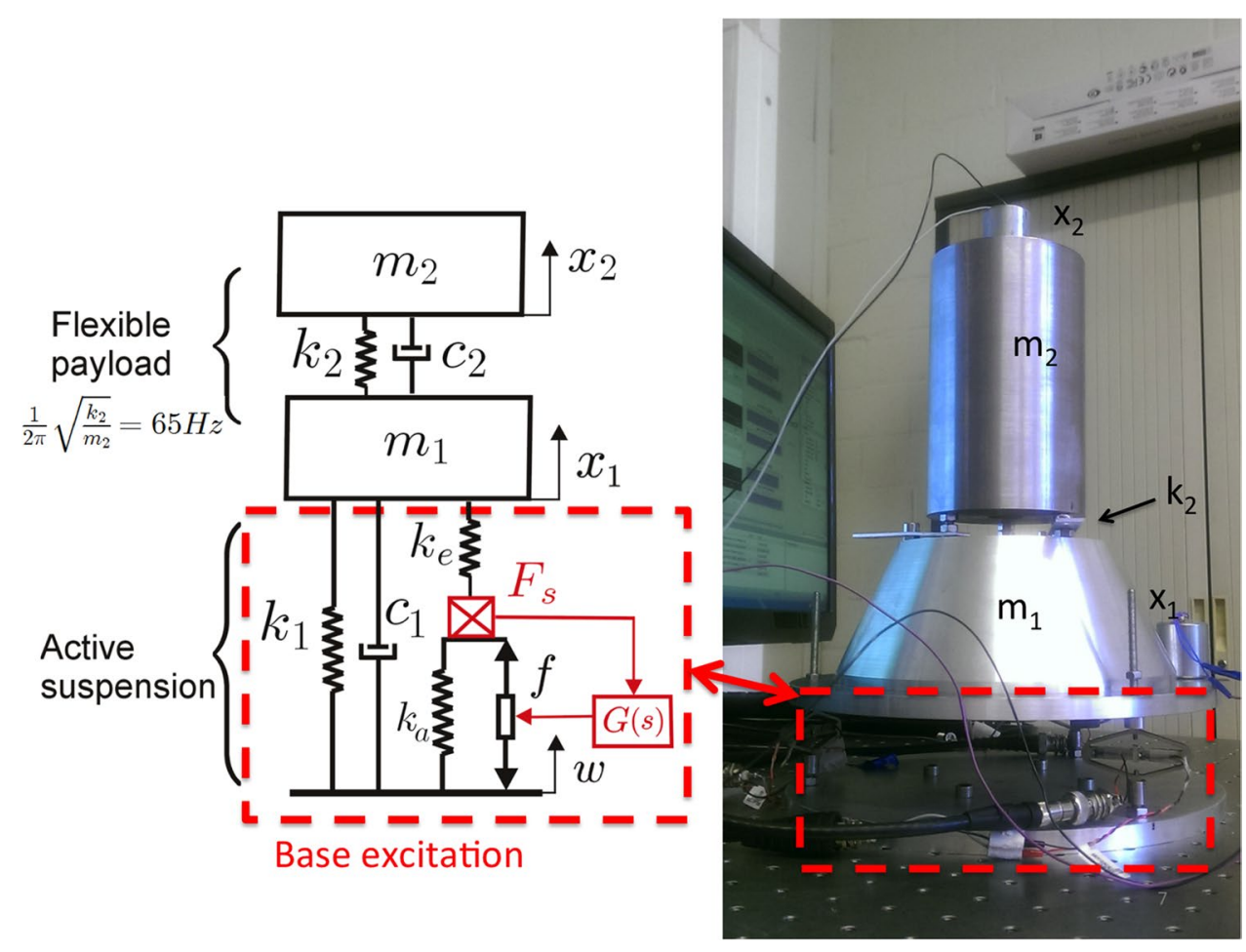

9. For both figures, the same quantities are shown: Top left: Transfer function between the shaker noise and one force sensor; Bottom left: integrated (downwards) RMS value of the transfer function between the shaker noise and one force sensor; Top right: transmissibility between the table top $w$ and $m_{2}$; bottom right: Transmissibility between the table top $\mathrm{w}$ and $m_{1}$. For both campaigns, one sees that both the suspension modes and the flexible modes of the payload can be critically damped. Furthermore, the fraction of the force transmitted to the payload that is measured by the force sensor is also significantly reduced by up to two orders of magnitude around the resonances, and the RMS value is reduced by a factor 5 . 
Fig. 7 Mode shapes and corresponding resonance frequencies of the first six modes
$17 \mathrm{~Hz}$

$57 \mathrm{~Hz}$
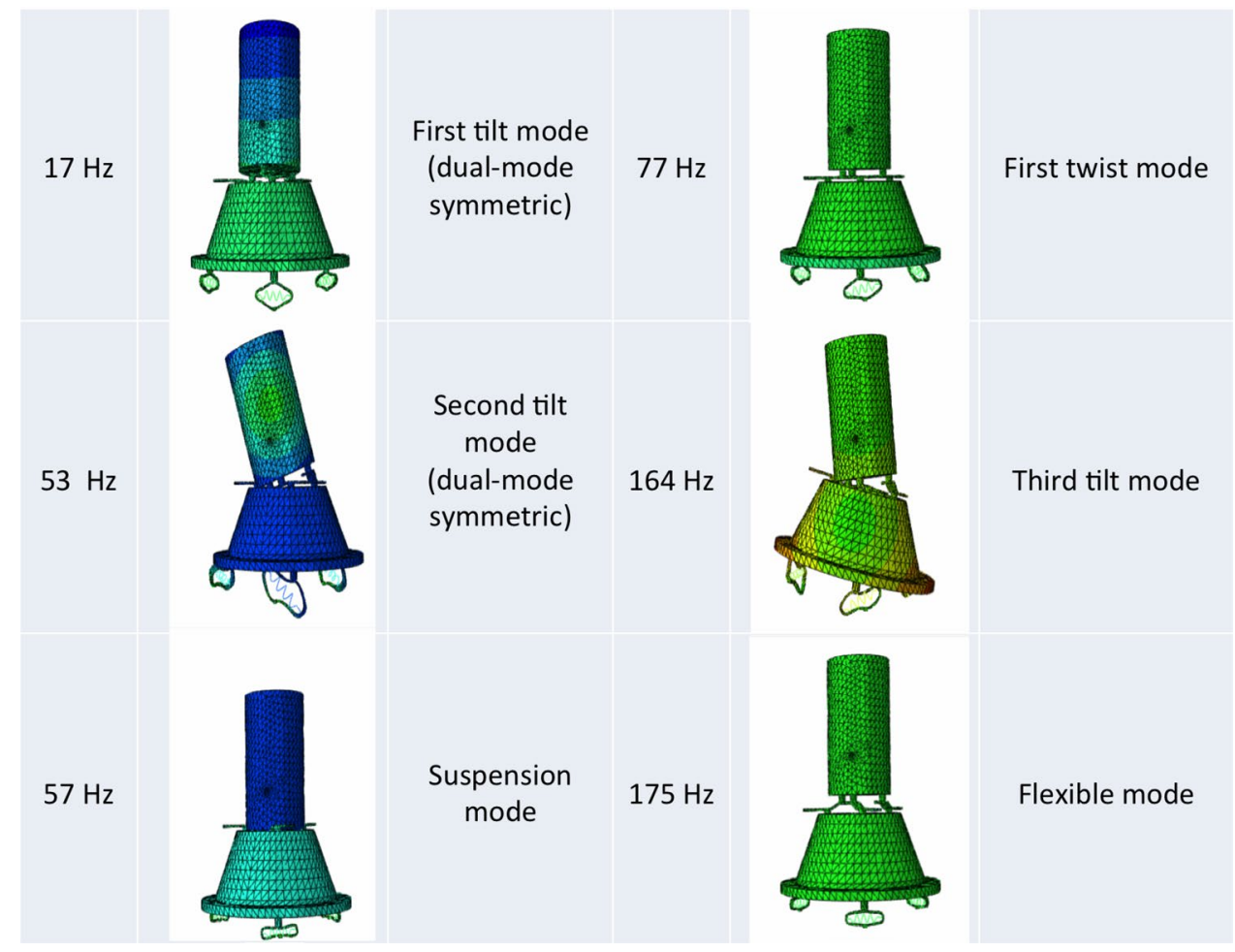
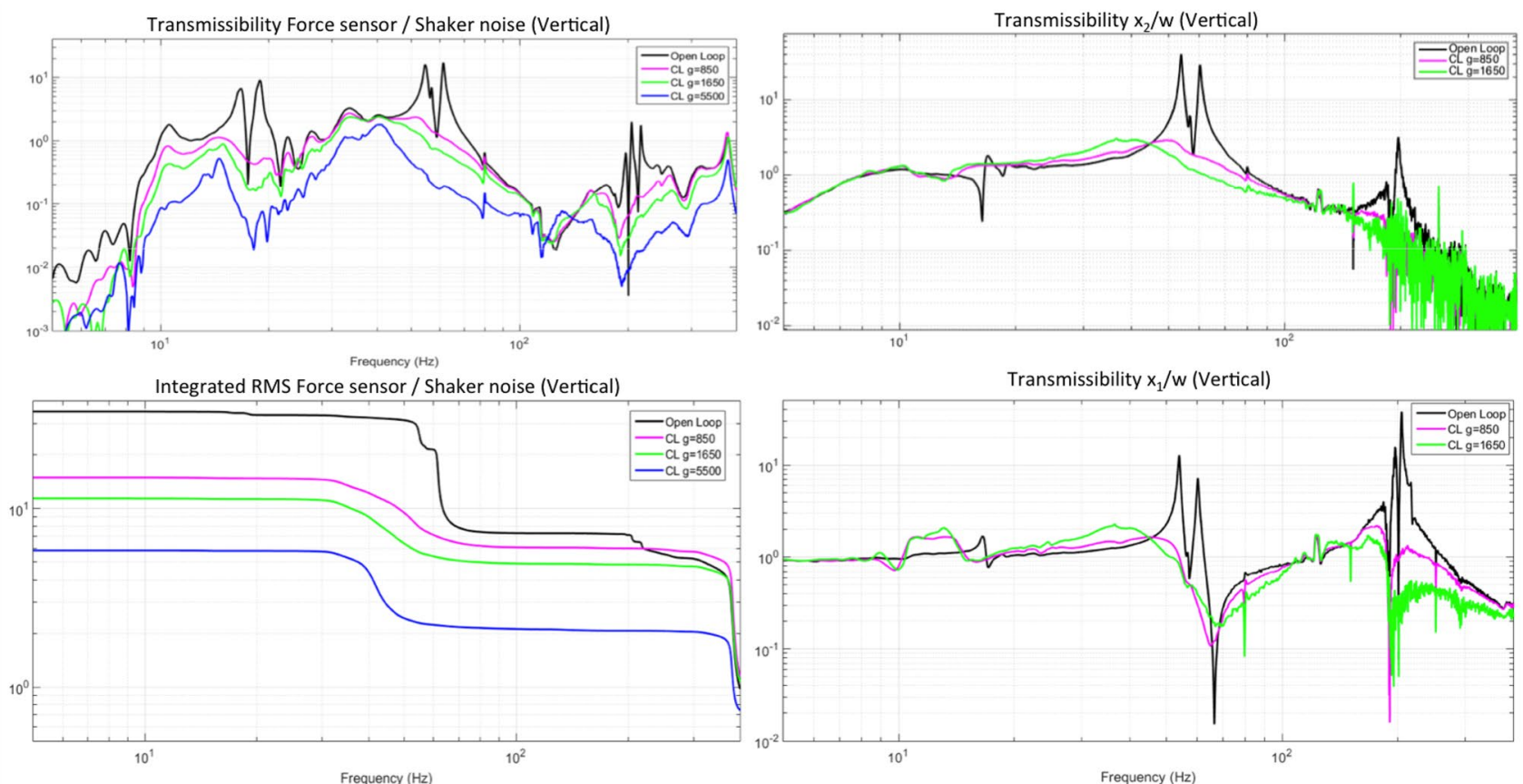

Fig. 8 Vertical excitation. Top left: transfer function between the shaker noise and one force sensor; bottom left: integrated (downwards) RMS value of the transfer function between the shaker noise

It has to be noticed that a degradation of the response is visible, mainly when excitation is horizontal. The origin of this behavior has not been investigated in detail, but may be

and one force sensor; top right: transmissibility between the table top $w$ and $m_{2}$; bottom right: transmissibility between the table top $w$ and $m_{1}$

due to an interaction of the tilt mode with the high pass filter used in the controller to cut the integration at low frequency in order to avoid saturations in the control signal. 

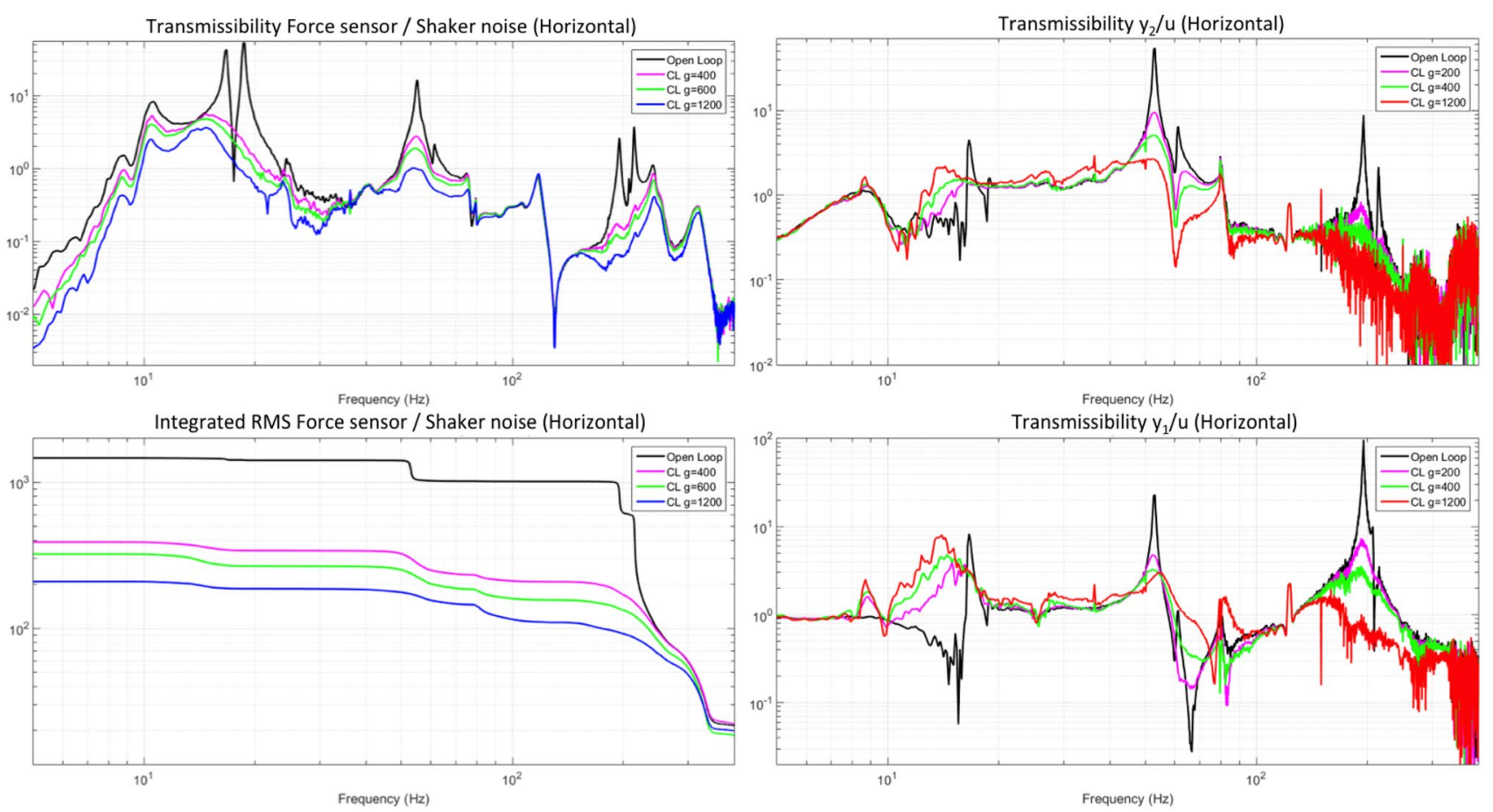

Fig. 9 Horizontal excitation. Top left: transfer function between the shaker noise and one force sensor; bottom left: integrated (downwards) RMS value of the transfer function between the shaker noise and one force sensor; top right: transmissibility between the horizon-

tal motion of the table top $(u)$ and horizontal motion of $m_{2}\left(y_{2}\right)$; bottom right: transmissibility between the horizontal motion of the table top $(u)$ and horizontal motion of $m_{1}\left(y_{1}\right)$
Fig. 10 Flexible payload mounted on a set of three isolators, including a flexible structure below the isolator: picture and simplified sketch showing only the vertical direction
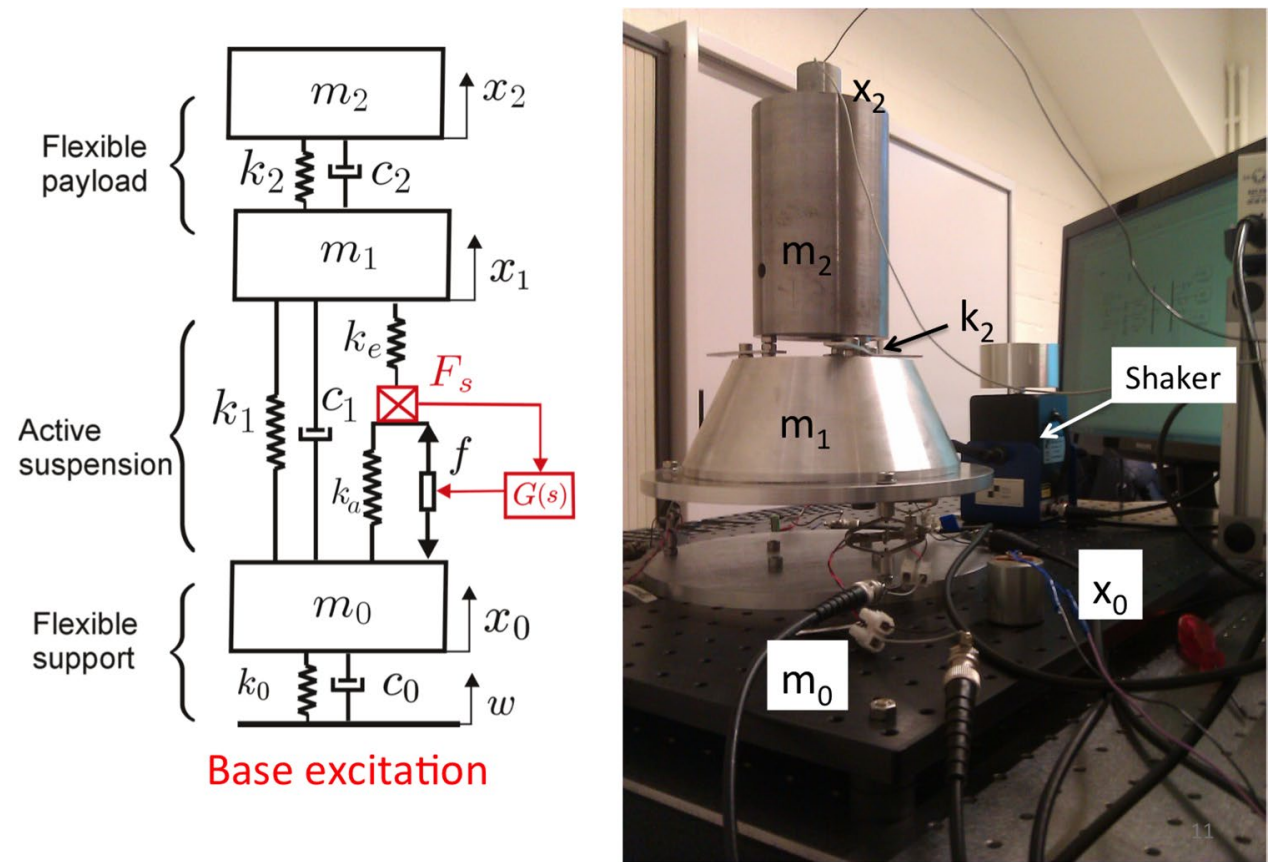

\section{Mounting on flexible structure}

The third setup considered in this study is shown in Fig. 10. It is the same as in the previous section, except that a flexible support has been introduced between the isolator and the table top. The flexible support consists of a steel plate $\left(m_{0}\right.$ in Fig. 10) mounted on four commercial rubber mounts. The objective of this experiment is to test the performance of 

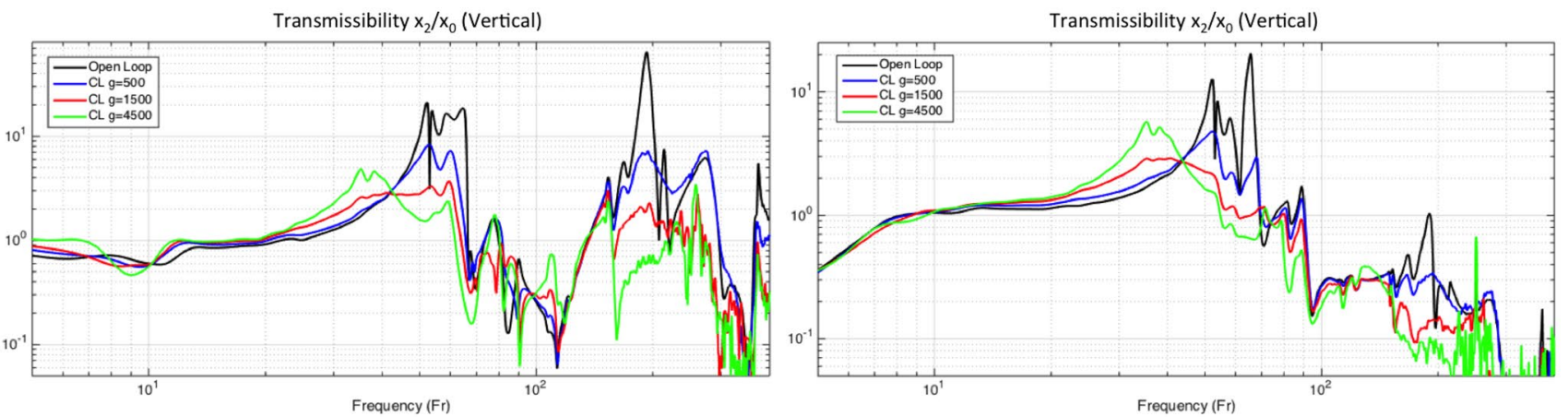

Fig. 11 Transmissibilities $x_{1} / x_{0}$ and $x_{2} / x_{0}$ between the flexible support and the payload for various values of the control gain, when the structure is excited in the vertical direction

the controller in the presence of flexibilities on both sides of the isolator.

The same experimental campaign has been conducted: base excitation in both the vertical and horizontal direction with a shaker mounted on the table top, as shown in Fig. 10. As an illustration, Fig. 11 shows the transmissibility between the flexible support and the payload for various values of the control gain, when the structure is excited in the vertical direction. This figure has to be compared with the right part of Fig. 8. Although the modal density is larger, one can still clearly identify the first and the second resonances, respectively, around 60 and $190 \mathrm{~Hz}$. The authority on these modes remains unchanged, and no stability issue has been encountered.

Similar results have been obtained for the excitation in the horizontal direction, and the reduction of the force transmission is also similar to those shown in Figs. 8 and 9.

\section{Conclusion}

A concept of active isolator for space payloads exposed to launch vibration has been proposed in this paper. The isolator comprises a metallic suspension and a pair of actuator and sensors collocated. The advantages of this concept are that the number and position can be adjusted easily and that it combines a high authority and a good robustness.

The concept has been tested experimentally using a commercial actuator on three setups of increasing complexity, ranging from a single d.o.f. payload to a flexible payload, isolated by three active mounts installed on flexible structures. For each setup, the controller has shown a high performance in reducing the peak responses of both suspension resonances and payload resonances, along with a broadband reduction of the force transmitted to the payload. Although the same controller has been used for the three setups, no stability issue has been encountered.
Future work in this activity will cover a design optimization of the flexures and refinement of the controller, e.g., including dedicated algorithms [16], and combining the active control with passive damping obtained by a resistive/inductive shunt in order to reduce the power consumption [17].

Acknowledgements The authors gratefully acknowledge the reviewers for their precious comments who lead to significant improvement of the quality of the manuscript. The F.R.S.-FNRS is warmly acknowledged for funding this research.

\section{References}

1. Chen, Y., Fang, B., Yang, T., Huang, W.: Study of whole-spacecraft vibration isolators based on reliability method. Chin. J. Aeronaut. 22(2), 153-159 (2009)

2. Johal, R., Christensen, J., Doud, D.: ORBCOMM generation 2 access to LEO on the Falcon 9 using SoftRide, a case history, AIAA/USU conference on small satellites (2012)

3. White, J., Fowler, E., Noble, K.: SoftRide ${ }^{\circledR}$ Shock and Vibration Isolation Systems, 2nd workshop on spacecraft shock environment and verification, ESA-ESTEC, Noordwijk, The Netherlands, 19-20th November 2015

4. Johnson, C.D., Wilke, P.S., Pendleton, S.C.: Softride vibration and shock isolation systems that protect spacecraft from launch dynamic environments. In: Proceedings of the 38th aerospace mechanisms symposium, Langley Research Center, 1-15 (2006)

5. Lan, W.D., Kaushish, V., Newman, J.H.: Progress in reducing vibration levels on the naval, Postgraduate School CubeSat Launcher (2015)

6. Collette, C., et al.: Review of active vibration isolation strategies. Recent Pat. Mech. Eng. 4, 212-219 (2011)

7. Chi, W., Cao, D., Huang, W.: Design of active whole-spacecraft vibration isolation based on voice-coil motor. SPIE smart structures and materials + nondestructive evaluation and health monitoring, 90613X-90613X (2014)

8. Thomas, G.R., Fadick, C.M., Fram, B.J.: Launch vehicle payload adapter design with vibration isolation features. In: 12th annual SPIE international symposium on smart structures and materials (2005) 
9. Rittweger, A., Beig, H.-G., Konstanzer, P., Dacal, R.B.: Feasibility demonstration of an active payload adaptor for Ariane 5. In: European conference on spacecraft structures (2005)

10. Strauch, H., Jetzschmann, C., Albus, J.: Robust controller design and testing of an active isolation for a payload-launcher system. In: 9th international ESA conference on guidance, navigation and control system, Porto, June 2-6 (2014)

11. Jean, P.: Isolation vibratoire par contrôle semi-actif d'amortisseurs magnéto-rhéologiques pour l'interface lanceur/charge utile, $\mathrm{PhD}$ thesis, Paris (2009)

12. Pierrick, J., Ohayon, R., Le Bihan, D.: Semi-active control using magnetorheological dampers for payload launch vibration isolation, smart structures and materials $61690 \mathrm{H}-61690 \mathrm{H}$ (2006)
13. Preumont, A., Dufour, J.P., Malekian, C.: Active damping by local force feedback with piezoelectric actuators. AIAA J. Guid. Control 15(2), 390-395 (1992)

14. Preumont, A.: Vibration Control of Active Structures: An Introduction, 3rd edn. Springer, Berlin (2011)

15. Preumont, A., François, A., Bossens, F., Abu-Hanieh, A.: Force feedback versus acceleration feedback in active vibration isolation. J. Sound Vib. 257, 605-613 (2002)

16. Chesné, S., Milhomem, A., Collette, C.: Enhanced damping of flexible structures using force feedback. AIAA J. Guid. Control Dyn. 39(7), 1654-1658 (2016)

17. Collette, C., Chesné, S.: Robust hybrid mass damper. J. Sound Vib. 375, 19-27 (2016) 